\title{
Fractionating Impulsivity: Contrasting Effects of Central 5-HT Depletion on Different Measures of Impulsive Behavior
}

\author{
Catharine A Winstanley*,', Jeffrey W Dalley', David EH Theobald' and Trevor W Robbins' \\ 'Department of Experimental Psychology, University of Cambridge, Cambridge, UK
}

\begin{abstract}
Reducing levels of 5-HT in the central nervous system has been associated with increases in impulsive behavior. However, the impulsivity construct describes a wide range of behaviors, including the inability to withhold a response, intolerance to delay of reward and perseveration of a nonrewarded response. Although these behaviors are generally studied using instrumental paradigms, impulsivity may also be reflected in simple Pavlovian tasks such as autoshaping and conditioned activity. This experiment aimed to characterize further the effects of central 5-HT depletion and to investigate whether different behavioral measures of impulsivity are inter-related, thus validating the construct. Rats received intracerebroventricular (ICV) infusions of vehicle $(n=10)$ or the serotonergic neurotoxin 5,7dihydroxytryptamine $(n=12)$ which depleted forebrain $5-\mathrm{HT}$ levels by about $90 \%$. Lesioned animals showed significant increases in the speed and number of responses made in autoshaping, increased premature responding on a simple visual attentional task, enhanced expression of locomotor activity conditioned to food presentation, yet no change in impulsive choice was observed, as measured by a delay-discounting paradigm. Significant positive correlations were found between responses made in autoshaping and the level of conditioned activity, indicating a possible common basis for these behaviors, yet no correlations were found between other behavioral measures. These data strengthen and extend the hypothesis that 5-HT depletion increases certain types of impulsive responding. However, not all measures of impulsivity appear to be uniformly affected by 5-HT depletion, or correlate with each other, supporting the suggestion that impulsivity is not a unitary construct.

Neuropsychopharmacology (2004) 29, I331-1343, advance online publication, 31 March 2004; doi: I 0.1038/sj.npp. I 300434
\end{abstract}

Keywords: serotonin; impulsivity; temporal discounting; Pavlovian conditioning; disinhibition; 5,7-DHT

\section{INTRODUCTION}

Impulsivity can be broadly defined as action without foresight, although the ability to act on impulse can sometimes be beneficial as well as detrimental. However, high levels of impulsivity are associated with attention deficit hyperactivity disorder (ADHD), mania, substance abuse, and personality disorders (DSM IV, 1994). Understanding the neural and neurochemical basis of impulsive behavior could therefore provide valuable insight into such psychiatric disorders. However, it has proved difficult to determine the exact nature of impulsivity. The application of factor analysis to self-report questionnaires assessing impulsiveness indicate that impulsive behavior consists of several independent dimensions, although there is considerable variation in the definition of these constituent

*Correspondence: CA Winstanley, Department of Experimental Psychology, University of Cambridge, Downing Street, Cambridge CB2 3EB, UK, Tel: + 441223333 550, Fax: + 441223333 564,

E-mail: caw42@cam.ac.uk

Received 27 August 2003; revised 19 December 2003; accepted 29 January 2004

Online publication: 9 February 2004 at http://www.acnp.org/citations/ Npp02090403391/default.pdf parts (Buss and Plomin, 1975; Dickman, 1990, 1993). Common themes include decreased inhibitory control, intolerance of delay to rewards, over-rapid decision-making due to lack of adequate reflection, as well as more universal deficits such as poor attentional ability.

Given the diversity of behavior that the term impulsivity appears to cover, it may be questioned as to what extent impulsivity is a unitary construct, as distinct from a term used to classify a variety of phenomena that may have independent underlying biological mechanisms (Evenden, 1999; Moeller et al, 2001). Through fractionating impulsivity into its different aspects, it is possible to devise operant behavioral tasks to measure these various forms of impulsive behavior in laboratory animals. These tasks can be broadly divided into those measuring impulsive decision making, or impulsive choice, and those measuring impulsive action, or behavioral disinhibition. The most successful example of the former category are delaydiscounting paradigms in which impulsive choice is defined as the selection of a small but immediate reward over a larger but delayed one (Evenden and Ryan, 1996; Mazur, 1987; Richards et al, 1999; Wogar et al, 1993). In the latter category, it has also proved possible to assess the ability of an animal to withhold from making a response, for example 
in differential reinforcement of low-rate schedules (DRL) (eg Fletcher, 1995; Richards et al, 1993), and in 'go/no-go' tasks (eg Harrison et al, 1999).

In support of the general hypothesis that decreased 5-HT function increases impulsive behavior (Linnoila et al, 1983; Soubrié, 1986), selective lesions of the 5-HT system in the rat have been reported to increase impulsivity on these measures (eg Fletcher, 1995; Harrison et al, 1997a, b, 1999; Mobini et al, 2000; Wogar et al, 1993). The finding that the same neurochemical manipulation increases all these aspects of impulsivity suggests that impulsivity is a unitary construct, at least in terms of its regulation by the serotonergic system. However, data from human volunteers following tryptophan depletion, which leads to decreases in levels of 5-HT in the CNS, have consistently found increases in impulsive action, but not in impulsive choice (Crean et al, 2002; LeMarquand et al, 1999, 1998). These data suggest that behavioral disinhibition, rather than impulsive decision-making, may be more sensitive to alterations in the serotonergic system. Furthermore, the increase in impulsive choice seen in delay-discounting tasks in rats following serotonergic lesions has not always been observed (Winstanley et al, 2003), nor found to be permanent (Bizot et al, 1999). Recently, a battery of tasks was used to measure impulsive behavior in a group of human volunteers, and no correlation was observed between the different indices of impulse control (McDonald et al, 2003), strongly suggesting that impulsivity is not underpinned by a unitary process. One of the aims of this investigation was therefore to apply a similar approach to the study of impulsivity in rodents, specifically by training the same cohort of animals on a variety of different tests of impulsive behavior.

The majority of tests designed to measure impulsivity in rats depend upon the response-dependent instrumental performance (eg delay-discounting, DRL schedules). However, behavior is also strongly influenced by associations formed through Pavlovian conditioning. For example, it is well-known that stimuli which are associated with addictive drugs are critically implicated in the maintenance and relapse of drug-seeking in humans and laboratory animals (Arroyo et al, 1998; Childress et al, 1992; Everitt and Robbins, 2000; Gawin, 1991), and subjects which exhibit greater tendencies to form and respond to Pavlovian stimulus-reward associations may be more vulnerable to substances of abuse (Tomie, 1996). Furthermore, it has been hypothesized that an increase in impulsive behavior plays a key role in the development and maintenance of drug addiction (Jentsch and Taylor, 1999; Volkow and Fowler, 2000), and increases in empirical measurements of impulsivity have been reported in substance abusers (Fillmore and Rush, 2002). Thus, it has been suggested that aspects of impulsivity may be present in behaviors elicited through Pavlovian conditioning procedures, such as autoshaping (Tomie et al, 1998a,b). Indeed, it has previously been reported that those animals which make more autoshaping responses show higher levels of impulsive choice in a delaydiscounting task (Tomie et al, 1998a).

In a typical version of the autoshaping paradigm designed for use with rats, subjects learn to associate the presentation of a visual stimulus (CS + ) with the delivery of reward, coming to approach the stimulus even though this action does not affect the reinforcement schedule, and therefore is not under the control of instrumental contingencies (Bussey et al, 1997). In a subsequent testing stage known as the negative automaintenance schedule, or omission phase, subjects continue to approach the CS + even though these responses are now punished by cancellation of the scheduled reward (Browne, 1976; Jenkins and Moore, 1973; Williams and Williams, 1969). Such behavior suggests that the animal is unable to withhold from making the motor response once it has become associated with food reward, thus reflecting impulsive action or 'motoric impulsivity'. Given the discussion above, autoshaping may therefore be affected by manipulations of the serotonergic system.

Serotonergic neurons are heavily implicated in the regulation of sensory and motor output (Jacobs et al, 2002). It is therefore perhaps unsurprising that impulsive action is modulated by a neurotransmitter system involved in the regulation of locomotor activity. Furthermore, it has been suggested that manipulations of the 5-HT system may influence emotional and cognitive behavior via indirect effects on more basic systems (Jacobs and Fornal, 1999), and that the serotonergic actions of psychotropic drugs contributes to their actions on motor, as well as affective and cognitive, symptoms of disorders such as schizophrenia and depression (Maes and Meltzer, 1995; Millan, 2000; Millan et al, 2003). Enhanced locomotor activity as observed in tests of exploratory behavior in novel environments, although traditionally thought to represent a decrease in anxiety, could also be thought of as an increase in behavioral disinhibition, and has more recently been described as an increase in impulsive behavior (eg Scearce-Levie et al, 1999). However, the effects of 5-HT lesions on tests of locomotor activity have provided ambiguous results, and it would appear that globally decreasing 5-HT only enhances motility if the environment is familiar to the animal, rather than novel (Gerson and Baldesserini, 1980).

One test of locomotor activity that may more accurately capture aspects of behavioral disinhibition is conditioned locomotor activity to food. In this test of Pavlovian conditioning, rats are fed at the same time each day and their locomotor activity monitored. An elevation in activity is typically observed prior to the anticipated delivery of food, indicating the formation of a Pavlovian association between the specific time of day and food delivery (Matthews et al, 1996). Given the suggestion outlined above that aspects of motoric impulsivity may be present in Pavlovian autoshaping tasks, likewise behavioral disinhibition as measured by conditioned locomotor activity to food may reflect impulsivity. Similar to autoshaping, the hyperactive response observed during the locomotor conditioning experiment does not affect the reinforcement contingency. However, in contrast to autoshaping, omission of the expected reward results in fairly rapid extinction of the conditioned response (Matthews et al, 1996).

Hence, through assessing the effect of ICV 5,7-DHT lesions on the acquisition and performance of a set of behavioral tasks reflecting different aspects of impulsive behavior, the aims of this study were as follows: (1) to observe which of these behavioral measures were affected by chronic 5-HT depletion; (2) to determine whether the serotonergic lesion altered performance of tasks based on 
Pavlovian conditioning, and if such changes could be interpreted as increases in behavioral disinhibition; (3) to determine the extent to which individual performance on these different tasks was intercorrelated, thus addressing the issue of whether impulsivity is a unitary construct. Each behavioral test was carried out in distinctive apparatus to minimize task interference.

\section{METHODS}

\section{Subjects}

Subjects were 22 male, Lister Hooded rats (Charles River, UK) weighing 300-320 g at the start of the experiment and were maintained on $14 \mathrm{~g}$ of rat chow per day. Water was available ad libitum. Animals were housed in pairs under a reverse light cycle (lights on from 1900 to 0700) and testing took place between 0900 and 13006 days per week. All experiments were carried out in strict accordance with the UK Animals (Scientific Procedures) Act 1986.

\section{Surgery}

Subjects were randomly divided into sham and lesion groups ( $n=10$ and 12 , respectively). All rats were treated $30 \mathrm{~min}$ before the start of surgery with $15 \mathrm{mg} / \mathrm{kg}$ desipramine $\mathrm{HCl}$ (Sigma Chemical Co., UK) dissolved in doubledistilled water to protect noradrenergic neurons from the neurotoxin. Rats were anesthetized with Avertin (10 g 2,2,2tribromoethanol, (Sigma, Poole, UK) in $5 \mathrm{~g}$ tertiary amyl alcohol, diluted in a solution of $40 \mathrm{ml}$ ethanol and $450 \mathrm{ml}$ PBS) given at a dose of $1 \mathrm{ml} / 100 \mathrm{~g}$ (i.p.), and secured in a stereotaxic frame fitted with atraumatic earbars. Rats in the lesion group received bilateral intracerebroventricular (ICV) infusions of $80 \mu \mathrm{g}$ (free base) 5,7-DHT creatinine sulfate (Sigma Chemical Co., UK) dissolved in $10 \mu \mathrm{l}$ of $0.1 \%$ ascorbic acid in saline, while the shams received bilateral ICV infusions of $10 \mu \mathrm{l}$ vehicle. Following each $8 \mathrm{~min}$ infusion, the injector was left in place for $2 \mathrm{~min}$ before withdrawal to allow the infusate to diffuse. The coordinates used were: AP $-0.9 \mathrm{~mm}$ from bregma, $\mathrm{L} \pm 1.5 \mathrm{~mm}$ from the midline, DV $-3.5 \mathrm{~mm}$ from dura, calculated from a stereotaxic atlas (Paxinos and Watson, 1998). The incisor bar was set at $-3.3 \mathrm{~mm}$ relative to the interaural line in a flat skull position. After surgery, animals were given free access to food for 10 days prior to behavioral testing to allow for the degeneration of serotonergic neurons (Bjorkland et al, 1975).

\section{Behavioral Testing}

All apparatus was controlled and monitored by software written in Arachnid, a real-time extension to BBC BASIC running on Acorn Archimedes Series computers (Cambridge, UK). All data were analyzed using SPSS version 9.0 (SPSS Inc., Chicago, IL, USA).

\section{Experiment 1: Autoshaping}

Behavioral apparatus. The autoshaping apparatus and behavioral training have been described previously in detail (Dalley et al, 2002a). Behavioral testing took place in six test chambers measuring $45 \times 32 \times 30 \mathrm{~cm}^{3}$ (Cambridge Cognition, Cambridge, UK) each housed within a soundattenuating wooden box fitted with a fan for ventilation and masking of extraneous noise, and illuminated by a centrally located $3 \mathrm{~W}$ houselight. The chambers were fitted with two food magazines and pellet dispensers which allowed the controlled delivery of $45 \mathrm{mg}$ sucrose pellets (Noyes dustless pellets, Sandown Scientific, UK). One food magazine was located at the rear of the box, directly above a pressure-sensitive floor panel. The chamber was fronted at one end with a video display unit (VDU: Intasolve Ltd, UK) upon which the stimuli were presented. A second food magazine was centrally located within a vertical chimney placed $6 \mathrm{~cm}$ in front of the VDU. The stimuli, consisting of vertical white rectangles $\left(10 \times 28 \mathrm{~cm}^{2}\right)$ were presented on either side of the chimney. Approaches to these stimuli could be detected by the breaking of photocell beams located to the right and to the left of the central magazine.

Pretraining. Subjects were initially given two $30 \mathrm{~min}$ habituation sessions to acclimatize to the test apparatus. During this time the houselight was on and pellets were delivered to the central magazine on a random time 40-s schedule. Pellets were also delivered to the second magazine at the rear of the chamber, but only following the depression of the pressure-sensitive floor panel, in order to shape animals to approach the back of the box. This food magazine was only used during pretraining.

Acquisition. On the day after pretraining, subjects were trained to associate a $10 \mathrm{~s}$ stimulus presented on the VDU with the delivery of a food pellet into the central magazine. A trial consisted of the consecutive presentation of the CS + and $\mathrm{CS}-$ in a randomized order. The CS + differed from the CS - in its spatial location (ie whether it appeared on the left or right of the food magazine) which was counterbalanced across subjects. Following a random time 10-40 s schedule (ISI - interstimulus interval), the rat was required to locate itself centrally on the floor panel at the rear of the chamber, thus eliminating chance approaches to the stimuli and ensuring equal stimulus sampling. Depression of the floor panel triggered the $10 \mathrm{~s}$ presentation of either the CS + or CS - . The offset of the CS + was contingent with the delivery of a food pellet in the central magazine, whereas the CS - was never associated with food reward. The delivery of food started another variable ISI, thereby separating the presentation of stimuli to minimize interference. The minimum time between presentations of the stimuli was $10 \mathrm{~s}$, and the maximum number of consecutive presentations of either the CS + or CS- was two. Acquisition was recorded over three consecutive days, the first two consisting of 33 trials and the last of 34, thereby giving 100 trials in total. Approaches were scored as the breaking of either the left or right photocell beam, and only the first approach was measured during the stimulus presentation, regardless of the direction of the approach. Approaches were only included in the analysis if they were made to the stimulus that was actually presented on that trial. The number of approaches to the CS + and CS - per block of 10 trials, as well as the latencies to make these approaches, were determined for statistical analysis. The difference score 
(number of approaches to $\mathrm{CS}+/$ number of approaches made to CS + and to CS-) was also calculated as an index of the level of discrimination learning established in each block.

Omission. The day after the final acquisition session, the task contingencies were altered such that approaches to the CS + prevented the delivery of a food pellet. The session consisted of 50 trials, and again only the first approach was scored.

Data analysis. Data were subjected to repeated measures analysis of variance (ANOVA) with BLOCK (10 levels) as a within-subjects factor and LESION (two levels; lesion and sham) as a between-subjects factor.

\section{Experiment 2: 'One-Choice' Visual Attentional Task}

Behavioral apparatus. A detailed description of the ninehole apparatus has been provided previously (Carli et al, 1983). Eight $25 \times 25 \times 25 \mathrm{~cm}^{3}$ nine-hole boxes (Cambridge Cognition, UK) were used, each contained within a ventilated and sound-attenuating chamber, and illuminated by a $3 \mathrm{~W}$ houselight. To summarize, animals were trained to make a nose-poke response into the middle hole in an array of five evenly spaced response holes set into the back wall of the operant chamber following brief illumination $(0.5 \mathrm{~s})$ of a stimulus light located therein. Nose-poke responses into the apertures could be detected by photocell beams located across the mouth. A food magazine was located in the middle of the opposite wall into which food pellets could be dispensed (Noyes dustless pellets, $45 \mathrm{mg}$; Sandown Scientific, UK). An infrared beam located horizontally across the entrance to the magazine allowed recording of entries into the magazine.

Behavioral training. A more detailed description of behavioral training has already been provided (Dalley et al, 2002b). In brief, animals received five to six sessions per week until a high level of stable performance was reached ( $\geqslant 80 \%$ accuracy $\leqslant 20 \%$ omissions). Each session consisted of 100 trials which had to be completed within $30 \mathrm{~min}$. The duration of the stimulus light was gradually reduced over 12 stages from $30 \mathrm{~s}$ in stage 1 to $0.5 \mathrm{~s}$ in stage 12 . A correct response was rewarded with a food pellet (Noyes dustless pellets, $25 \mathrm{mg}$; Sandown Scientific, Middlesex, UK) whereas an incorrect, premature or lack of response was punished by nondelivery of reward and a $5 \mathrm{~s}$ time-out during which the houselight was extinguished. Repeated responding during the presentation of the light stimulus or during the limited hold period was classified as perseverative responding and, while monitored, was not punished.

Data analysis. Seven variables were analyzed: the percent of correct responses made ((number of correct responses + number of incorrect responses/total correct and incorrect responses) $\times 100$ ); percent of responses omitted ((number of omissions/total number of correct, incorrect and omitted responses $) \times 100)$; percent of premature responses ((number of premature responses/total number of trials) $\times 100$ ), latency to make a correct response, latency to collect reward, perseverative responses and the total number of trials completed per session. Variables which were expressed as a percentage were subjected to an arcsine transformation in order to limit the effect of an artificially imposed ceiling (ie 100\%). Performance during acquisition of the task was assessed through repeated measures ANOVA of each variable, with STAGE (12 levels) as a within-subjects factor and LESION (two levels, sham and lesion) as a between-subjects factor. Stable performance following acquisition of the task was determined by ANOVA with one within-subjects factor, DAY (seven daily test sessions) and one between-subjects factor, LESION (two levels, sham and lesion).

\section{Experiment 3: Conditioned Locomotor Activity}

Apparatus. Locomotor activity was assessed in individual activity cages $\left(25 \times 40 \times 18 \mathrm{~cm}^{3}\right)$, each with two photocell beams located $1 \mathrm{~cm}$ above the floor at either end of the cage.

Pre-exposure. Activity tests of $90 \mathrm{~min}$ duration were given at the same time each day (between 1500 and 1800) until stable levels of activity were reached. Water was available ad libitum in the activity cages. Animals were fed at varying and unpredictable times between 2 and $4 \mathrm{~h}$ after the activity tests.

Conditioning. Feeding was switched from the home cages to the photocell activity cages. After half an hour of the 90 min activity test, the animals' daily allowance of food $(14 \mathrm{~g})$ was placed in a plastic bowl inside the activity chamber. Animals were not fed again in the home cage. This continued for 12 days.

Prefeeding. To test the resilience of the conditioned activity to the incentive value of the food, animals were given free access to food for $1 \mathrm{~h}$ prior to being placed in the activity cages. Animals were still fed in the activity cages after half an hour as in the conditioning sessions.

Data analysis. Locomotor activity in the $30 \mathrm{~min}$ prior to food delivery, as measured by the total number of beam breaks recorded, was assessed through ANOVA with one between-subjects factor (SESSION, 12 levels) and one between-subjects factor (LESION, two levels, sham and lesion). In keeping with a previous study (Matthews et al, 1996), data were square root transformed to reduce inhomogeneity of variance, as recommended by Howell (1997).

\section{Experiment 4: Delay-Discounting}

Behavioral apparatus. The apparatus has been described in more detail previously (Cardinal et al, 2000). Testing occurred in a set of eight operant chambers (Med Associates Inc., USA), each enclosed within a ventilated and soundattenuating wooden box. The front aluminum wall of each chamber was fitted with two retractable levers. Centrally located between the two levers was a food magazine into which $45 \mathrm{mg}$ food pellets could be delivered (Noyes dustless pellets, Sandown Scientific, UK). Entry to the food magazine could be detected by the breaking of an infrared photobeam located horizontally across the entrance. 
General illumination was provided by a $2.8 \mathrm{~W}$ houselight mounted on the rear aluminum wall of the chamber.

Pretraining. Subjects were first trained under an FR1 schedule to a criterion of 50 presses in $30 \mathrm{~min}$, first for the left lever, then for the right. The depression of a lever and the delivery of food was accompanied by a 'click' sound produced by an electronic noise generator ('clicker'). Subsequently, subjects were trained to make a nose-poke response in the central food magazine which triggered presentation of a lever. Every $40 \mathrm{~s}$, a trial began with illumination of the houselight and traylight. Failure to make a response in the food magazine or subsequently to depress the lever within $10 \mathrm{~s}$ terminated the trial and the houselight was turned off until the next trial was scheduled to begin. A response on either lever led to the delivery of one food pellet. In every pair of trials, the left lever was presented once and the right once, through the order within the pair of trials was random. Rats were trained to a criterion of 60 successful trials in $1 \mathrm{~h}$.

Behavioral procedure. Throughout the task, response on one lever (lever A) would produce a reward of one pellet (reward A) whereas a response on the other (lever B) would produce a reward of four pellets (reward B). The position of these levers (left or right) was kept constant for each rat, but was counterbalanced between rats. Each session consisted of 60 trials which started at $60 \mathrm{~s}$ intervals. Within each session, 30 trials were forced choice (15 of lever A and 15 of lever B) and 30 free choice (ie) both levers were presented. A high proportion of forced-choice trials were included to try and reduce the influence of side bias on choice behavior. Each session was divided into five identical blocks of 12 trials, the first six of which would be pairs of forced choice trials where each pair consisted of one presentation of lever $A$ and one of lever B in a random order. The last six would then be free choice trials.

The start of each trial was signaled by the illumination of the houselight and traylight. As in training, a nose-poke response was required in the food magazine to trigger lever presentation, and failure to make a response in the food magazine or subsequently to depress the lever within $10 \mathrm{~s}$ terminated the trial. When either lever was pressed, the houselight was turned off, the 'clicker' sounded, and both levers were retracted. Food reward was then delivered, accompanied by a 'click' noise and the onset of the traylight, either almost immediately or after a delay. When the food was collected, the traylight was switched off and the chamber returned to the ITI state. The experiment was divided into five sets of five sessions. During the first set, delivery of either the small or large rewards was virtually immediate. The delay to reward $A(\mathrm{~d} A)$ remained constant at $0 \mathrm{~s}$ throughout the entire experiment, whereas the delay to reward $B(\mathrm{~d} B)$ increased to $10 \mathrm{~s}$ in set $2,20 \mathrm{~s}$ in set 3 , and $40 \mathrm{~s}$ in set 4 . Set 5 was identical to set 1 (ie) $\mathrm{d} A$ and $\mathrm{d} B$ were both $0 \mathrm{~s}$.

Data analysis. Only the data from the last two sessions of each set were analyzed. The data were subjected to a repeated measure ANOVA, with DELAY (four levels: 0, 10, 20 , and $40 \mathrm{~s}$ ) as a within-subjects factor and LESION (two levels, sham and lesion) as a between subjects factor.

\section{Correlational Analysis}

The presence of any correlations between performance of the different tasks was also assessed. The data from each rat on each measure or interest were ranked, and the ranked data analyzed through bivariate correlations using Kendall's tau as the correlation coefficient. The following behavioral measures were selected from each task: the total number of approaches made during acquisition of autoshaping (totalapp) and during omission (totalom), the total number of approaches made to the CS + during acquisition of autoshaping (cs_app) and during omission (cs_om), the average latency to approach the CS + during autoshaping (cs_lat) and during omission (cs_latom), the average premature responses made during stable performance of the simple attentional task (prem); the percentage increase in locomotor activity from baseline observed during the final session of conditioning locomotor activity to food (condloco), and during the first session of prefeeding (prfloco); the rate of delay discounting as determined by the gradient of the line formed through plotting the number of choice of the large reward against the delay to the large reward (grad) and the indifference point at which subjects chose the large reward $50 \%$ of the time, obtained from this line (indif).

\section{Ex Vivo Lesion Analysis}

At the end of the experiment, animals were killed through exposure to increasing concentrations of carbon dioxide. The brains were then rapidly removed and frozen on dry ice. Thereafter, coronal sections were cut ( $150 \mu \mathrm{m}$ thickness) on a cryostat $\left(-10^{\circ} \mathrm{C}\right)$ from the frontal pole and mounted onto prechilled microscope slides. A stainless-steel micropunch $(0.75 \mathrm{~mm}$ diameter) was used to remove $0.3-0.6 \mathrm{mg}$ aliquots of tissue from the following (left and right) brain regions: nucleus accumbens, prelimbic cortex, anterior cingulate cortex, dorsomedial striatum, dorsolateral striatum, amygdala, ventral hippocampus, dorsal hippocampus, septum, and hypothalamus. Samples were homogenized in $75 \mu \mathrm{l}$ of $0.2 \mathrm{M}$ perchloric acid to precipitate protein material. Following centrifugation at $6000 \mathrm{rpm}$ for $20 \mathrm{~min}$ at $4^{\circ} \mathrm{C}, 50 \mu \mathrm{l}$ of the supernatant was decanted and placed into autoinjector microvials ready for analysis. Levels of dopamine (DA), noradrenaline (NA), serotonin (5-HT), and 5-hydroxyindoleacetic acid (5-HIAA) were determined in brain samples by reversed-phase high-performance liquid chromatography (HPLC), as described previously (Matthews et al, 2001).

\section{RESULTS}

\section{Lesion Assessment}

In keeping with previous reports (eg Harrison et al, 1997a, b; Winstanley et al, 2003), postmortem analyses of 5-HT concentrations throughout the forebrain revealed a statistically significant reduction in 5-HT levels in lesioned animals as compared to sham-operated controls of over $90 \%$ in all regions assayed (see Table 1). Levels of 5-HIAA were also significantly reduced. Levels of DA, DOPAC, and NA were not significantly affected. 


\section{Experiment 1: Autoshaping}

Results from the analysis of the number of approaches made to the visual stimuli in each block of 10 trials (Figure 1a) demonstrate that both sham-operated and lesioned animals developed discriminated approach behavior over repeated exposure to the reinforcement contingencies, approaching the $\mathrm{CS}+$ more than the $\mathrm{CS}-$ (STIMULUS: $\mathrm{F}_{1,20}=5.233, . p<0.033$; BLOCK: $\mathrm{F}_{9,180}=7.904$, $p<0.0001$; BLOCK $\times$ STIMULUS: $\mathrm{F}_{9,180}=3.512, p<0.0001$ ). Overall, there was a highly significant main effect of the lesion (LESION: $\mathrm{F}_{1,20}=20.684, p<0.001$ ), as 5-HT-depleted animals made significantly more approaches to both the $\mathrm{CS}+$ and CS-.

However, as shown in Figure 1c, despite demonstrating a greater number and speed of conditioned responses, the lesioned animals exhibited the same level of discrimination between the CS + and the CS- as the sham-operated controls, as indicated by analysis of the difference scores calculated for each block (LESION: $\mathrm{F}_{1,20}=0.021$, NS; BLOCK $\times$ LESION: $\mathrm{F}_{9,180}=1.095$, NS).

Analysis of the approach latencies revealed that the latency to approach the CS + decreased as training progressed (BLOCK: $\mathrm{F}_{9,180}=4.651, p<0.0001$ ). In addition, lesioned animals were significantly faster to approach the $\mathrm{CS}+$ than the sham controls (Figure 1d, BLOCK $\times$ LESION: $\mathrm{F}_{9,180}=2.632, p<0.034$, LESION: $\mathrm{F}_{1,20}=7.893, p<0.011$ ). The corresponding analysis of latencies to approach the CS - was confounded due to the low numbers of responses made by the sham animals, therefore these results are not reported.

During the omission phase, where approaches to the $\mathrm{CS}+$ now lead to cancellation of reward, ICV 5,7-DHT lesioned animals continued to make significantly more

Table I Tissue Concentrations of 5-HT, 5-HIAA, DA, DOPAC, and NA in Cortical, Striatal, and Limbic Areas of ICV 5,7-DHT Lesioned and Sham-Operated Rats

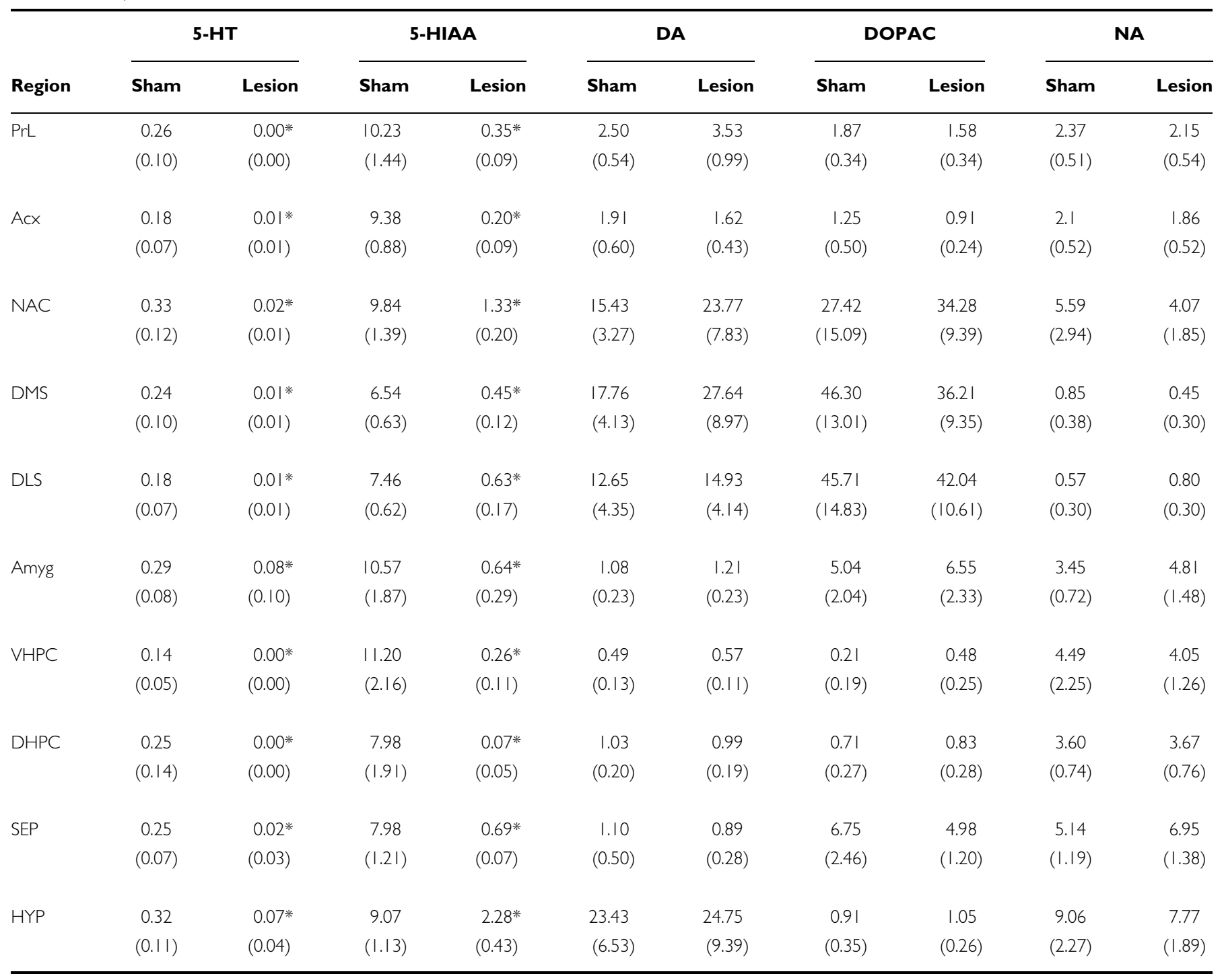




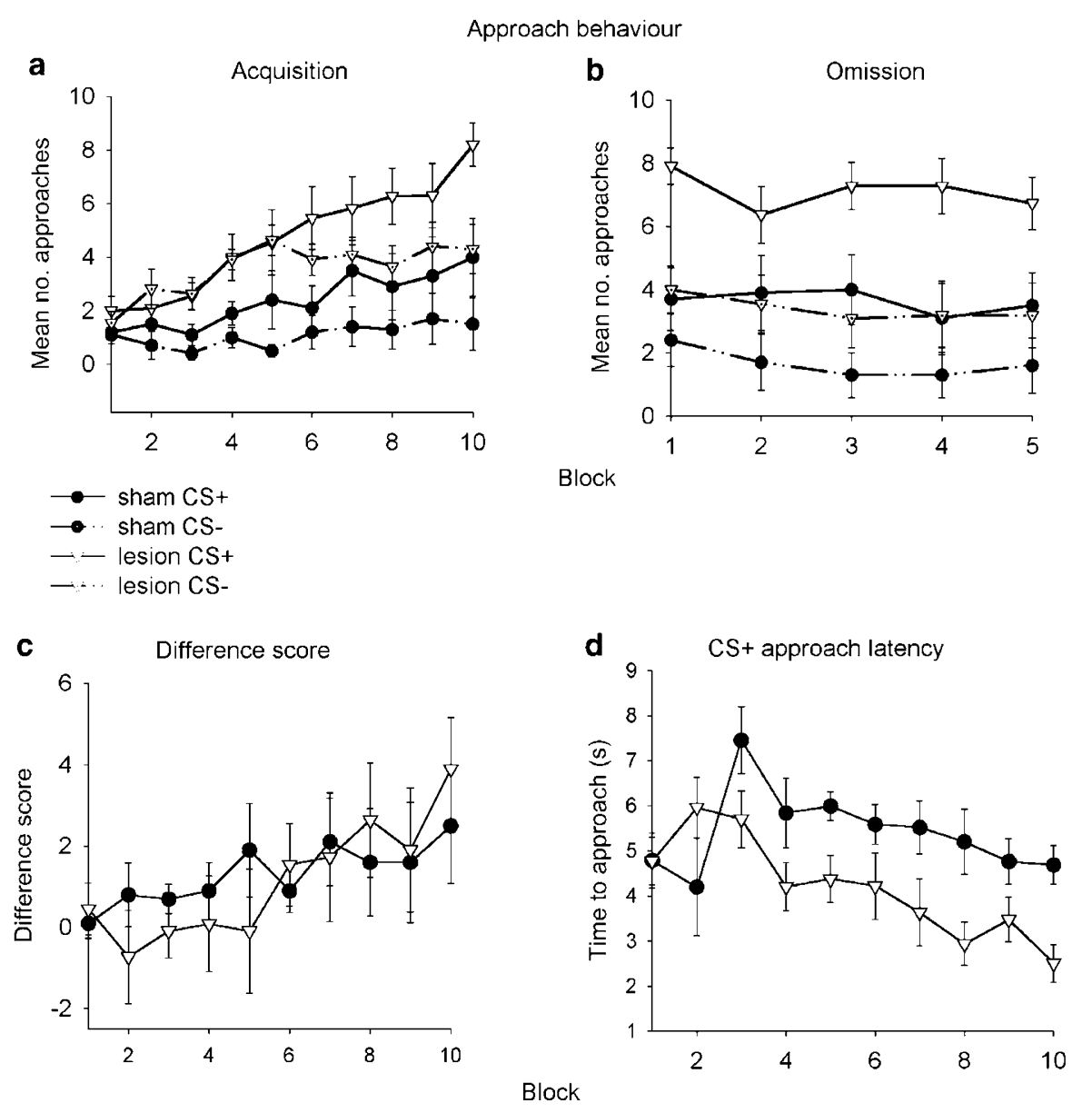

Figure I Effects of ICV 5,7-DHT lesions on different behavioral measures during autoshaping: (a) responses to the CS + and CS - during acquisition; (b) responses to the CS + and CS- during the omission phase; (c) the difference scores calculated for each block during acquisition; (d) the latency to approach the CS + during acquisition. Data shown are mean and SEM.

approaches to the CS $+\left(\operatorname{LESION}_{1,19}=8.986, p<0.007\right)$ but not to the CS- $\left(\operatorname{LESION}_{1,19}=2.571, \mathrm{NS}\right)$ in comparison to sham-operated controls (Figure 1b). As in acquisition of the autoshaping behavior, lesioned animals were also faster to approach the CS + , but this effect fell short of significance (mean latency to approach CS + \pm SEM: sham $4.44 \mathrm{~s} \pm 0.69 ; \quad$ lesion $3.27 \pm 0.47 ; \quad \mathrm{F}_{1,20}=3.556$, $p<0.074)$.

\section{Experiment 2: 'One-Choice’ Visual Attentional Task}

Both sham-operated and lesioned animals acquired this task over a similar number of sessions (mean number of sessions \pm SEM: sham $28.1 \pm 0.58$; lesion $27.5 \pm 1.05$ ). Throughout acquisition, lesioned animals made significantly more premature responses compared to sham controls (Figure 2a, LESION: $\mathrm{F}_{1,20}=5.264, p<0.033$ ). Lesioned animals continued to show elevated levels of premature responding once they had acquired stable baseline performance of the task (Figure 2b, LESION: $\left.F_{1,20}=6.468, p<0.019\right)$. In contrast, no other variable, such as accuracy (Figure $2 \mathrm{c}$ and $\mathrm{d}$ ), was significantly affected by the lesion during acquisition (LESION $\mathrm{F}_{1,20}=1.615, \mathrm{NS}$ ) or performance (LESION: $\left.\mathrm{F}_{1,20}=2.638, \mathrm{NS}\right)$ of the task.

\section{Experiment 3: Conditioned Locomotor Activity}

There were no differences between the sham-operated and lesioned animals' levels of locomotor activity following habituation to the locomotor cages prior to the onset of the conditioning procedure (LESION: $\mathrm{F}_{1,17}=2.650$, NS). During conditioning, levels of locomotor activity significantly increased in both lesioned animals (SESSION $\mathrm{F}_{8,64}=3.249$, $p<0.032$ ) and in sham controls (SESSION: $\mathrm{F}_{8,64}=7.856$, $p<0.003$; Figure 3a). However, the increase in activity was significantly more pronounced across the conditioning sessions in lesioned animals (LESION: $\mathrm{F}_{1,17}=5.115$, $p<0.037, \quad$ SESSION $\times$ LESION $\left.=\mathrm{F}_{8,136}=4.30, \quad p<0.011\right)$. Further analysis comparing levels of activity during each conditioning session between lesioned animals and shamoperated controls revealed that lesioned animals demonstrated potentiated conditioned locomotor activity from conditioning session 5 onwards (independent samples $t$-test, $t=-2.425, p<0.027)$.

Prefeeding significantly decreased locomotor activity in both sham controls (SESSION: $\mathrm{F}_{1,8}=88.321, p<0.001$ ) and lesioned animals (SESSION: $\mathrm{F}_{1,8}=43.216, p<0.0001$ ) compared to locomotor activity recorded in the last session of conditioning (Figure $3 \mathrm{~b}$ ). Subsequent to prefeeding, locomotor activity in the sham group had returned to a level comparable to that recorded prior to conditioning 
PREMATURE RESPONSES

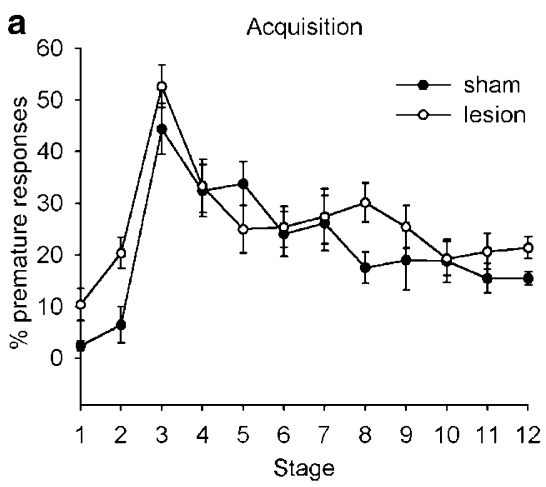

b

b Performance

c

Acquisition

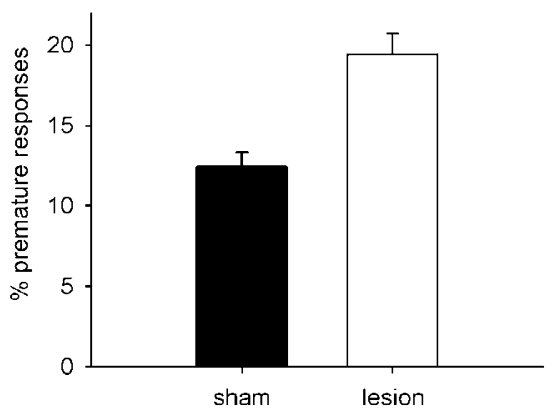

d Performance
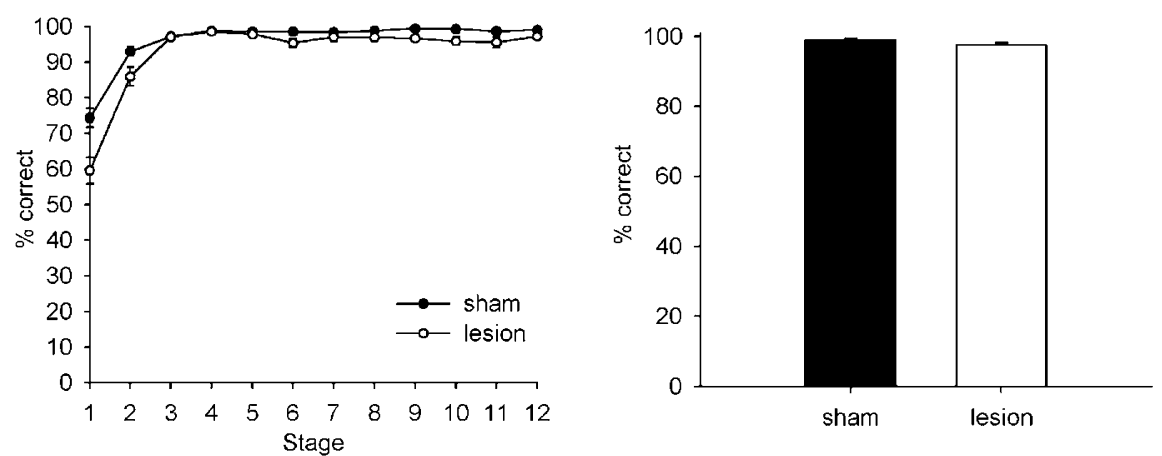

Figure 2 Effects of ICV 5,7-DHT lesions on the number of premature responses made during acquisition (a) and performance (b), and the percent of correct responses made during acquisition (c) and performance (d), of a simple visual attentional task. Data shown are mean and SEM.

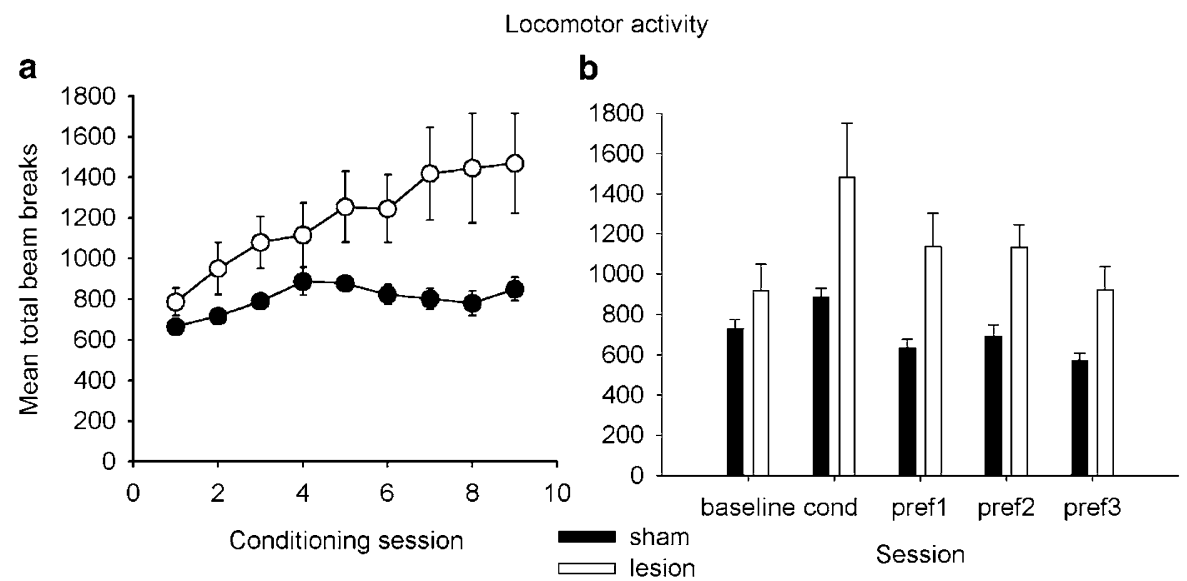

Figure 3 Effects of ICV 5,7-DHT lesions on the acquisition of conditioned locomotor activity (a) and the effects of prefeeding prior to the conditioning sessions (b). Abbreviations: cond, final conditioning session; prefl-3, prefeeding sessions I-3. Data shown are mean and SEM.

(SESSION: $\mathrm{F}_{1,8}=0.661, \mathrm{NS}$ ), but lesioned animals still demonstrated higher levels of locomotor activity than they did during baseline testing (SESSION $\times$ LESION: $\mathrm{F}_{1,16}=14.310, p<0.002$; lesioned animals only SESSION: $\left.\mathrm{F}_{1,8}=20.982, p<0.002\right)$. The prefeeding manipulation was repeated another two times until the locomotor activity of the lesioned animals returned to baseline levels (third prefeeding day, SESSION: $\left.\mathrm{F}_{1,8}=1.210, \mathrm{NS}\right)$.

\section{Experiment 4: Delay-Discounting}

Both sham-operated and lesioned animals chose the large reward on almost every trial when the delay to the large reward was $0 \mathrm{~s}$ (Figure 4). As the delay to the large reward increased, the preference of both groups of rats shifted towards the smaller but more immediate reward (DELAY: $\left.\mathrm{F}_{3,48}=45.404, p<0.0001\right)$. When the delay to the large 


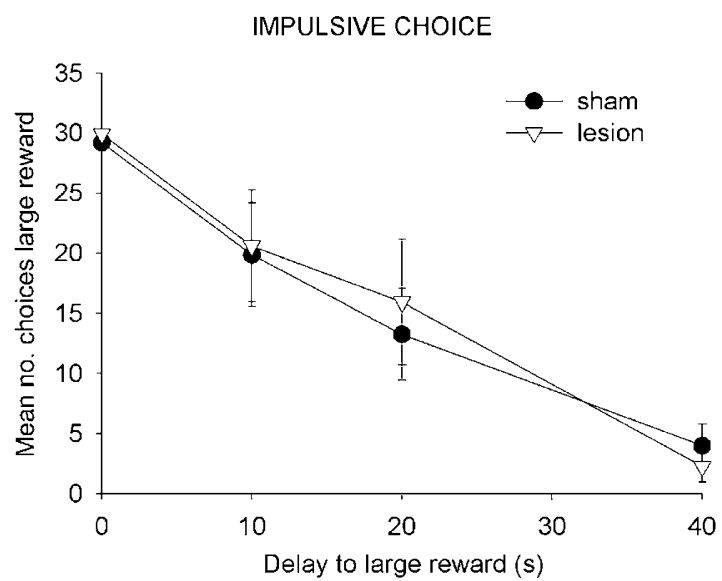

Figure 4 Lack of effect of ICV 5,7-DHT lesions on delay-discounting. Data shown are mean and SEM.

reward was removed in the final set of sessions, both sham and lesioned animals returned to choosing the large reward on nearly all trials. However, there was no significant effect of the ICV 5,7-DHT lesion on choice behavior at the different delays (LESION: $\mathrm{F}_{1,16}=0.013$, NS; DELAY $\times$ LESION: $\left.\mathrm{F}_{3,48}=0.304, \mathrm{NS}\right)$.

\section{Retraining on 'One-Choice' Visual Attentional Task}

As this negative result was obtained with the final behavioral paradigm used, the animals were retrained on the simple visual attentional task to determine whether the 5-HT depletion was still effective in altering behavior. As before, a significant increase in premature responses was observed of a comparable magnitude to that observed in Experiment 2 (mean number of premature responses \pm SEM: sham 20.24 \pm 3.36 ; lesion $27.83 \pm 3.98$; LESION $_{1,15}=$ 5.061, $p<0.04)$.

\section{Correlational Analysis}

Owing to the large number of correlations made, only those which were significant at the $p<0.01$ level are emphasized. As shown in Table 2, the majority of the measures taken from the same task were highly correlated with each other. The only significant correlation observed between the putative measures of impulsivity obtained from different tasks was between the levels of conditioned activity expressed to food and the total number of approaches made during the omission phase of autoshaping (condloco $v s$ total om: $\tau=0.543, p<0.0001)$ and the number of approaches made to the CS during the omission phase (condloco vs cs_om: $\tau=0.413, p<0.007$ ). In order to observe whether the lesioned animals were driving the correlation in any way, the same analysis was performed on the control animals independently. However, the correlational analysis is weakened by dividing the cohort into sham and lesioned groups due to the smaller numbers of subjects in each division, and may therefore be less accurate. Nevertheless, when the lesioned animals were excluded from the analysis, the correlations between the autoshaping and conditioned activity measures remained significant at the $p<0.05$ level (condloco $v$ s total om: $\tau=0.584$., $p<0.02$; condloco $v s$ cs_om: $\tau=0.600, p<0.016$ ), and an additional significant correlation was observed between the total number of approaches made in the acquisition phase, and the level of conditioned locomotor activity (condloco $v s$ totalapp: $\tau=0.733, p<0.003$ ). These results suggest that the correlation between individual's performance during autoshaping and conditioned locomotor activity was not driven solely by the behavior of the ICV 5,7-DHT lesioned animals on these two tasks, despite the fact the lesion profoundly affect both behavioral measures.

\section{DISCUSSION}

This study extends previous work investigating the role of 5 -HT in impulsivity, and is the first to evaluate the effects of global 5-HT depletion in the same cohort of rats on a battery of tasks, thus enabling the measurement of different aspects of impulsive behavior within the same subjects. ICV 5,7-DHT lesions increased the number and speed of autoshaping responses, increased the number of premature responses made on the 'one-choice' visual attentional task, increased the expression of locomotor activity conditioned to food presentation, but did not alter impulsive choice on a delay-discounting task. This pattern of results suggests that global 5-HT depletion increased behavioral disinhibition or impulsive action, but not impulsive choice. Although the level of 5-HT depletion is long-lasting, it could be argued that the lack of effect on the delay-discounting task can be explained by some functional recovery in the CNS, as this paradigm was the last task in the battery. However, a similar increase in premature responses was still observed in lesioned animals when retested on the 'one-choice' task, indicating that global 5-HT depletion led to enduring and persistent effects. There was little correlation between the different indices of impulsivity obtained from the different tasks, supporting the view that impulsivity is not a unitary construct. Evidently, aspects of impulsivity can be differentially affected by neurochemical interventions, further indicating that different forms of impulsivity may be underpinned by different neurobiological mechanisms.

In keeping with data obtained following tryptophan depletion in human volunteers (Crean et al, 2002), global 5-HT depletion alone had no effect on delay-discounting. This contrasts with previous studies reporting increased choice of the small, immediate reward following serotonergic lesions of the dorsal and median raphé nuclei (Mobini et al, 2000; Wogar et al, 1993). Although the reasons for this discrepancy are unclear, there are a number of obvious differences between these studies, not least the use of different behavioral tasks, varying methodology, and the presence or absence of neuroprotective pretreatment strategies prior to surgery (a more detailed discussion of these factors is given in Winstanley et al, 2003). In addition, different effects of the two lesions (ICV vs intra-raphé infusions of 5,7-DHT) have previously been observed on different behavioral tests, for example, amphetamine selfadministration (Fletcher et al, 1999; Lyness et al, 1980), despite comparable levels of 5-HT depletion.

The conclusion that depleting 5-HT does not affect impulsive choice could be somewhat premature as only 
Table 2 Nonparametric Correlations Between Different Measures of Impulsivity

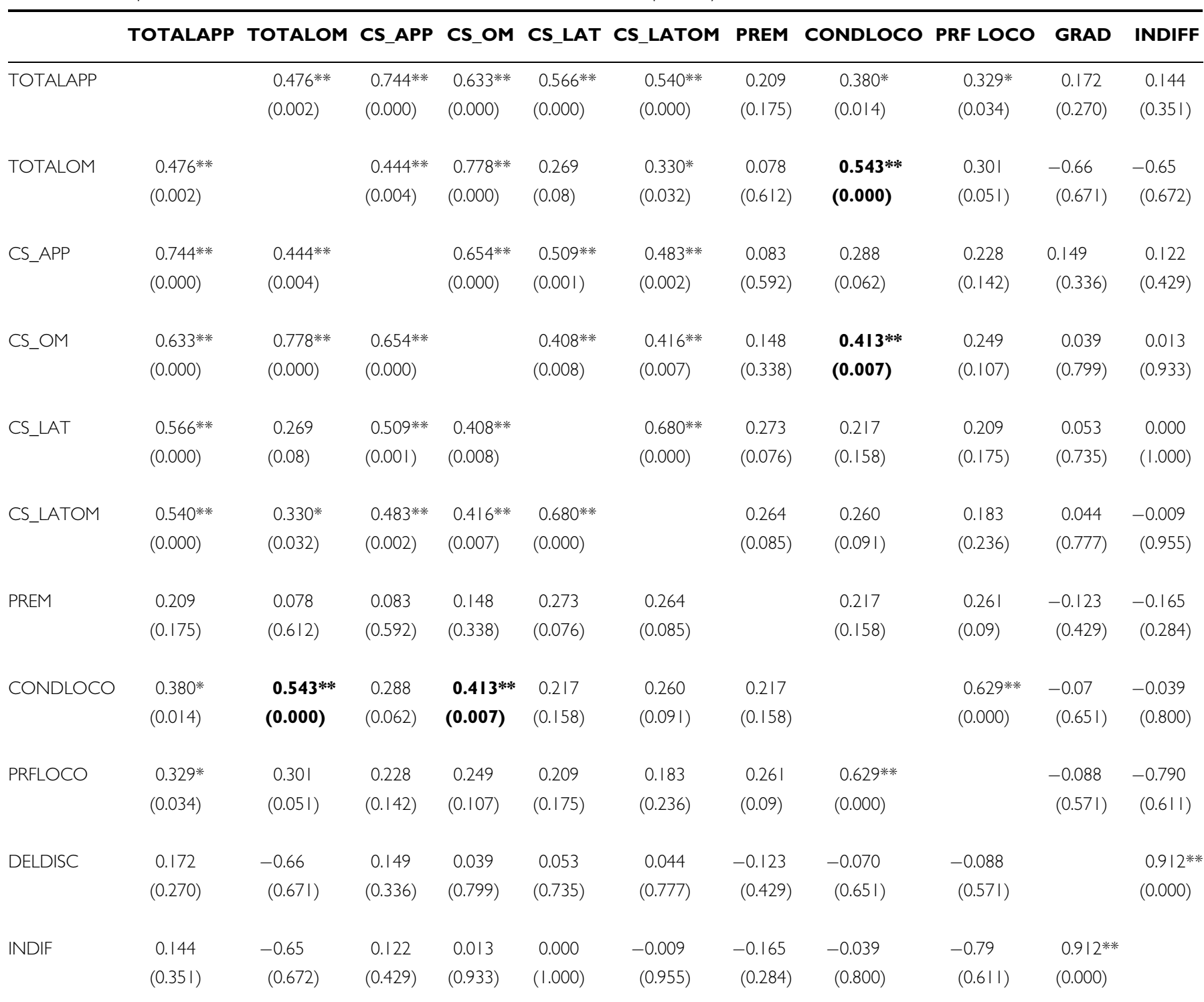

Data shown are Kendall's $\tau$ ( $p$-value). $*=p<0.05, * *=p<0.0$ I. Correlations between performance measures from different tasks which were significant at the $p<0.0$ l level are indicated by bold type.

one measure of impulsive choice (delay-discounting) was included in the task battery. However, in support of this suggestion, lesioning the serotonergic system does not affect probability discounting in rats, where subjects must choose between a small but certain $v s$ a larger but less certain reward (Mobini et al, 2000). In each of the tasks affected by serotonergic lesions in this study, the change in behavior could be viewed as an inability to withhold from responding during a period of anticipation, either of the reward or of stimuli signaling reward. In contrast to the other tasks utilized, the response providing the index of impulsivity in the delay-discounting paradigm does not occur during such a specific anticipatory phase. It would be of interest to observe whether global 5-HT depletion alters performance of delay-discounting tasks if behavior during the delay period, when anticipation of reward is high, could affect the reinforcement contingency. For example, the level of impulsive choice shown by pigeons altered if they were allowed to 'opt out' of their decision to choose the delayed reward once the delay had begun (Rachlin and Green, 1972).

The 5-HT system is also heavily implicated in timing processes (Ho et al, 2002), and a perceived 'speeding up' of the passage of time could theoretically elevate the number of premature responses made on the 'one-choice' task. However, lesions to the serotonergic system do not prevent accurate timing behavior per se, but impair temporal judgment when animals are required to regulate their own behavior in time (see Ho et al, 2002 for a comprehensive review). In the 'one-choice' task, animals are instructed when to respond by the onset of the stimulus light; therefore, 5-HT lesions may not be expected to produce a timing deficit. However, it is possible that alterations in timing processes may affect the response to food delivery in the conditioned locomotor activity paradigm, as there are 
no explicit cues signaling the arrival of food. Nevertheless, the time between the start of the locomotor testing and food delivery remains constant throughout the experiment, therefore the subjective interpretation of the length of that interval may not be relevant to the level of conditioned anticipatory activity.

One of the aims of this study was to determine whether autoshaping and conditioned locomotor activity paradigms can measure aspects of impulsivity. The fact that the ICV 5,7-DHT lesioned rats made more responses, and were quicker to do so, than sham-operated controls in the autoshaping paradigm, yet did not show any alterations in their ability to discriminate the CS + over the control stimulus, is suggestive of a deficit in inhibitory response control, particularly as lesioned subjects continued to make more responses to the CS + during the omission phase of the experiment, when doing so resulted in the cancellation of reward. It has often been suggested that 5-HT lesioned rats are less sensitive to punishment and cues-predictive of punishment, and this literature was originally used to support the serotonergic hypothesis of impulsivity. For example, Soubrie (1986) argued that the decrease in the level of conditioned suppression observed following decreases in central 5-HT levels was indicative of an inability to withhold from responding regardless of the consequences, and thus may have reflected impulsive tendencies.

The enhancement of autoshaping behavior and conditioned locomotor activity observed here following ICV 5,7DHT lesions may also be interpreted as an elevation in motivation for reward, particularly as serotonergic lesions have also been shown to increase responding for reward under progressive ratio schedules (Loh and Roberts, 1990; Roberts et al, 1994). It is therefore difficult to determine whether the increase in responding in such paradigms is due to increased motivation for reward, or a decrease in the ability to withhold from responding. However, it is possible to argue that any increase in impulsivity may be due to an elevated desire for reward that overrides the necessary requirement to inhibit responding, and it has even been suggested that a similar mechanism underpins the impulsive behavioral deficits observed in children with ADHD (Solanto, 1998). The finding that ICV 5,7-DHT lesioned animals were not spontaneously hyperactive, but did increase hypermotility in anticipation of the delivery of reward in the conditioned locomotor activity to food paradigm, indicates that the elevations in motor output resulting from 5-HT depletion are specific to situations where reward is expected. Whether such findings should be interpreted as a confound inherent in any test of impulsivity, or illustrative of one of the driving forces behind deficits in impulse control, is an interesting issue which may warrant further investigation.

The lack of correlation observed between performance on the delay-discounting and autoshaping tasks contradicts a previous report demonstrating that increased levels of impulsive choice were associated with increased autoshaping performance (Tomie et al, 1998a). The correlation observed in the Tomie et al study may have been confounded by the fact that both tasks involved lever-press responses and took place in very similar operant chambers, whereas care was taken in this study to minimize any interference effects arising from similarities of operant response or setting. Nevertheless, two of the indices of behavioral disinhibition examined were positively correlated in this study, namely the level of conditioned locomotor activity and the conditioned approaches made during autoshaping, yet premature responding in the onechoice task, another form of impulsive action, was not correlated with any other. An important behavioral distinction between the measurements of impulsivity obtained from the Pavlovian tests and the 'one-choice' task is that in the latter, the impulsive response is punished immediately, whereas in the former, impulsive responding is either unpunished (conditioned locomotor activity) or the punishment is delayed until the offset of the CS + (omission phase of autoshaping). The presence or absence of feedback following impulsive behavior may be an important factor in the pattern of correlations observed.

In light of the suggested explanation for the correlation observed in the Tomie et al, study, it is necessary to note that the responses made in both the autoshaping and conditioned activity tasks were essentially locomotor in nature, whereas in both the 'one-choice' and delaydiscounting tasks, subjects had to make a specific operant response on a lever or in the response aperture. This similarity in the motor repertoire could therefore be reflected in the correlation observed between these behavioral measures. However, the context in which these locomotor responses were made were very different in the two tasks. In autoshaping, subjects had to discriminate between two stimuli, only one of which was predictive of reward, and selectively approach this CS + , whereas conditioned locomotor activity involved nondirectional ambulation, linked to the formation of simpler associations between the time of day, the delivery of food and a familiar context.

Therefore, although the task battery included multiple measures of impulsive action, which were all heavily affected by the serotonergic lesion in a manner consistent with enhanced behavioral disinhibition, the relative lack of intraindividual correlation between the performance measures suggests that serotonergic modulation affected different aspects of impulsivity. Furthermore, decreasing 5-HT levels did not increase impulsive choice. These data provide direct preclinical evidence for the hypothesis that impulsivity is a nonunitary concept, which is not underpinned by a single neurobiological mechanism (Evenden, 1999; Moeller et al, 2001). In support of this suggestion, little correlation has been found between different measures of impulsivity in normal human volunteers (McDonald et al, 2003), and although clinical studies often use the Barratt Impulsiveness Scale (BIS) as an overall measure of 'trait impulsivity', factor analysis reveals that the items within this scale cluster into three independent factors (Patton et al, 1995). In a recent study using human volunteers, errors of commission on a continuous performance test (a potential measure of impulsive action analogous to the 5CSRT and 'one-choice' tasks) were strongly correlated with BIS scores, whereas performance on a delay-discounting task showed only a moderate correlation with this questionnaire measure (Swann et al, 2002). It has also been suggested that two sub-types of ADHD exist which have different 
neurobiological bases, and which are predominantly categorized by different measures of impulsivity; poor inhibitory control is associated with disordered thought and action regulated by mesocortical dopamine systems, and intolerance to delay of gratification reflects an altered motivational style linked to mesolimbic dopamine systems (Sonuga-Barke, 2002). Such data illustrate the utility of fractionating impulsivity to aid the understanding of behavioral disorders.

In summary, global 5-HT depletion enhanced impulsive action, but had no effect on impulsive choice in a battery of tasks measuring different aspects of impulsivity. By using a number of different tasks to investigate impulsive behavior rather than a single paradigm, it has been possible to categorize aspects of impulsivity, not only in terms of their susceptibility to disruption by neurochemical manipulations, but also using correlations between individuals' performance on the different measures. This strategy may allow a clearer understanding of the brain regions and neurotransmitter systems implicated in the control and regulation of impulsive behavior. Such information may improve the classification and treatment of neuropsychiatric disorders such as ADHD and mania which encompass a variety of deficits in impulse control.

\section{ACKNOWLEDGEMENTS}

This work was supported by a Wellcome Trust Programme grant and completed within the MRC Centre for Behavioral and Clinical Neuroscience. CAW was supported by an MRC Studentship.

\section{REFERENCES}

American Psychiatric Association Diagnostic and Statistical Manual IV (1994) American Psychiatric Association: Washington DC.

Arroyo M, Markou A, Robbins TW, Everitt BJ (1998). Acquisition, maintenance and reinstatement of intravenous cocaine selfadministration under second-order schedule of reinforcement in rats: effects of conditioned cues and continuous to cocaine. Psychopharmacology 140: 331-344.

Bizot JC, Le Bihan C, Puech AJ, Hamon M, Thiébot MH (1999). Serotonin and tolerance to delay of reward in rats. Psychopharmacology 146: 400-412.

Bjorkland A, Baumgarten HD, Rensch A (1975). 5,7-Dihydroxytryptamine: improvement of its selectivity for serotonin neurons in the CNS by treatment with desipramine. J Neurochem 24: 833-835.

Browne MP (1976). The role of primary reinforcement and overt movements in auto-shaping in the pigeon. Anim Learn Behav 4: 287-292.

Buss AH, Plomin R (1975). A Temperament Theory of Personality Development. Wiley: New York.

Bussey TJ, Everitt BJ, Robbins TW (1997). Dissociable effects of cingulate and medial frontal cortex lesions on stimulus-reward learning using a novel Pavlovian autoshaping procedure for the rat: implications for the neurobiology of emotion. Behav Neurosci 111: 908-919.

Cardinal RN, Robbins TW, Everitt BJ (2000). The effects of D-amphetamine, chlordiazepoxide, alpha- flupenthixol and behavioural manipulations on choice of signalled and unsignalled delayed reinforcement in rats. Psychopharmacology 152: $362-375$.
Carli M, Robbins TW, Evenden JL, Everitt BJ (1983). Effects of lesions to ascending noradrenergic neurons on performance of a 5-choice serial reaction time task in rats-implications for theories of dorsal noradrenergic bundle function based on selective attention and arousal. Behav Brain Res 9: 361-380.

Childress AR, Ehrman R, Roohsenow DJ, Robbins SJ, O’Brien CP (1992). Classically conditioned factors in drug-dependence. In: Lowinson W, Luiz P, Millman RB, Langard JG (eds). Substance Abuse: A Comprehensive Textbook. Williams \& Wilkins: Baltimore, USA.

Crean J, Richards JB, de Wit H (2002). Effect of tryptophan depletion on impulsive behavior in men with or without a family history of alcoholism. Behav Brain Res 136: 349-357.

Dalley JW, Chudasama Y, Theobald DE, Pettifer CL, Fletcher CM, Robbins TW (2002a). Nucleus accumbens dopamine and discriminated approach learning: interactive effects of 6-hydroxydopamine lesions and systemic apomorphine administration. Psychopharmacology 161: 425-433.

Dalley JW, Theobald DE, Eagle DM, Passetti F, Robbins TW (2002b). Deficits in impulse control associated with tonicallyelevated serotonergic function in rat prefrontal cortex. Neuropsychopharmacology 26: 716-728.

Dickman SJ (1990). Functional and dysfunctional impulsivity: personality traits and cognitive correlates. J Pers Soc Psychol 58: 95-102.

Dickman SJ (1993). Impulsivity and information processing. In: McCown WG, Johnson JL, Shure MB (eds). The Impulsive Client: Theory, Research and Rreatment. American Psychological Society: Washington, DC. pp 151-184.

Evenden JL (1999). Varieties of impulsivity. Psychopharmacology 146: 348-361.

Evenden JL, Ryan CN (1996). The pharmacology of impulsive behaviour in rats: the effects of drugs on response choice with varying delays of reinforcement. Psychopharmacology 128: 161-170.

Everitt BJ, Robbins TW (2000). Second-order schedules of drug reinforcement in rats and monkeys: measurement of reinforcing efficacy. Psychopharmacology 153: 17-20.

Fillmore MT, Rush CR (2002). Impaired inhibitory control of behavior in chronic cocaine users. Drug Alcohol Depend 66: 265-273.

Fletcher PJ (1995). Effects of combined or separate 5,7-dihydroxytryptamine lesions of the dorsal and median raphe nuclei on responding maintained by a DRL 20s schedule of food reinforcement. Brain Res 675: 45-54.

Fletcher PJ, Korth KM, Chambers JW (1999). Depletion of brain serotonin following intra-raphe injections of 5,7-dihydroxytryptamine does not alter $d$-amphetamine self-administration across different schedule and access conditions. Psychopharmacology 146: 185-193.

Gawin FH (1991). Cocaine addiction: psychology and neurophysiology. Science 250: 1580-1586.

Gerson SC, Baldesserini RJ (1980). Motor effects of serotonin in the central nervous system. Life Sci 27: 1435-1451.

Harrison AA, Everitt BJ, Robbins TW (1997a). Central 5-HT depletion enhances impulsive responding without affecting the accuracy of attentional performance: interactions with dopaminergic mechanisms. Psychopharmacology 133: 329-342.

Harrison AA, Everitt BJ, Robbins TW (1997b). Doubly dissociable effects of median- and dorsal-raphe lesions on the performance of the five-choice serial reaction time test of attention in rats. Behav Brain Res 89: 135-149.

Harrison AA, Everitt BJ, Robbins TW (1999). Central serotonin depletion impairs both the acquisition and performance of a symmetrically reinforced go/no-go conditional visual discrimination. Behav Brain Res 100: 99-112. 
Ho M-Y, Velazquez-Martinez DN, Bradshaw CM, Szabadi E (2002). 5-Hydroxytryptamine and interval timing behaviour. Pharmacol Biochem Behav 71: 773-785.

Howell DC (1997). Statistical Methods for Psychology. Wadsworth Publishing Company: Belmont, CA.

Jacobs BL, Fornal CA (1999). Activity of serotonergic neurons in behaving animals. Neuropsychopharmacology 21: 9S-15S.

Jacobs BL, Martin-Cora FJ, Fornal CA (2002). Activity of medullary serotonergic neurons in freely moving animals. Brain Res Brain Res Rev 40: 45-52.

Jenkins HM, Moore BR (1973). The form of the auto-shaped response with food or water reinforcers. Anim Learn Behav 20: 163-181.

Jentsch JD, Taylor JR (1999). Impulsivity resulting from frontostriatal dysfunction in drug abuse: implications for the control of behavior by reward-related stimuli. Psychopharmacology 146: 373-390.

LeMarquand DG, Benkelfat C, Pihl RO, Palmour RM, Young SN (1999). Behavioral disinhibition induced by tryptophan depletion in nonalcoholic young men with multigenerational family histories of paternal alcoholism. Am J Psychiatry 156: 1771-1779.

LeMarquand DG, Pihl RO, Young SN, Tremblay RE, Seguin JR et al (1998). Tryptophan depletion, executive functions, and disinhibition in aggressive, adolescent males. Neuropsychopharmacology 19: 333-341.

Linnoila M, Virkkunen M, Scheinin M, Nuutila A, Rimon R, Goodwin FK (1983). Low cerebrospinal-fluid 5-hydroxyindoleacetic acid concentration differentiates impulsive from nonimpulsive violent behavior. Life Sci 33: 2609-2614.

Loh EA, Roberts DC (1990). Break-points on a progressive ratio schedule reinforced by intravenous cocaine increase following depletion of forebrain serotonin. Psychopharmacology 101: 262-266.

Lyness WH, Friedle NM, Moore KE (1980). Increased selfadministration of D-amphetamine after destruction of 5-hydroxytryptaminergic nerves. Pharmacol Biochem Behav 12: 937-941.

Maes M, Meltzer HY (1995). The serotonin hypothesis of major depression. In: Bloom FE, Kupfer DJ (eds). Psychopharmacology: The Fourth Generation of Progress. Raven Press: New York. pp 933-944

Matthews K, Dalley JW, Matthews C, Tsai T-H, Robbins TW (2001). Periodic maternal separation of neonatal rats produces regional and gender-specific effects on biogenic amine content in post-mortem adult brain. Synapse 40: 1-10.

Matthews K, Scott Hall F, Wilkinson LS, Robbins TW (1996). Retarded acquisition and reduced expression of conditioned locomotor activity in adult rats following repeated early maternal separation: effects of prefeeding, $d$-amphetamine, dopamine antagonists and clonidine. Psychopharmacology 126: 75-84.

Mazur J (1987). An adjusting procedure for studying delayed reinforcement. In: Commons ML, Nevin JA, Rachlin HC (eds). Quantitative Analyses of Behaviour: The Effect of Delay and Intervening Events on Reinforcement Value. Erlbaum: Hillsdale, NJ. pp 55-73.

McDonald J, Schleifer L, Richards JB, de Wit H (2003). Effects of THC on behavioral measures of impulsivity in humans. Neuropsychopharmacology 28: 1356-1365.

Millan MJ (2000). Improving the treatment of schizophrenia: focus on serotonin (5-HT)(1A) receptors. J Pharmacol Exp Ther 295: 853-861.

Millan MJ, Veiga S, Girardon S, Brocco M (2003). Blockade of serotonin $5-\mathrm{HT}_{1 \mathrm{~B}}$ and $5-\mathrm{HT}_{2 \mathrm{~A}}$ receptors suppresses the induction of locomotor activity by 5 -HT reuptake inhibitors, citalopram and fluvoxamine, in NMRI mice exposed to a novel environment: a comparison to other 5-HT receptor subtypes. Psychopharmacology 168: 397-409.

Mobini S, Chiang TJ, Ho MY, Bradshaw CM, Szabadi E (2000). Effects of central 5-hydroxytryptamine depletion on sensitivity to delayed and probabilistic reinforcement. Psychopharmacology 152: 390-397.

Moeller FG, Barratt ES, Dougherty DM, Schmitz JM, Swann AC (2001). Psychiatric aspects of impulsivity. Am J Psychiatry 158: 1783-1793.

Patton JH, Stanford MS, Barratt ES (1995). Factor structure of the Barrat Impulsiveness Scale. J Clin Psychol 51: 768-774.

Paxinos G, Watson C (1998). The Rat Brain in Stereotaxic Co-ordinates. Academic Press: Sydney.

Rachlin H, Green L (1972). Commitment, choice and self-control. J Exp Anal Behav 17: 15-22.

Richards JB, Sabol KE, de Wit H (1999). Effects of methamphetamine on the adjusting amount procedure, a model of impulsive behavior in rats. Psychopharmacology 146: 432-439.

Richards JB, Sabol KE, Seiden LS (1993). DRL interresponse-time distributions: quantification by peak deviation analysis. $J$ Exp Anal Behav 60: 361-385.

Roberts DC, Loh EA, Baker GB, Vickers G (1994). Lesions of central serotonin systems affect responding on a progressive ratio schedule reinforced either by intravenous cocaine or by food. Pharmacol Biochem Behav 49: 177-182.

Scearce-Levie K, Chen J-P, Gardner E, Hen R (1999). 5-HT receptor knockout mice: pharmacological tools or models of psychiatric disorders. Ann NY Acad Sci 868: 701-715.

Solanto MV (1998). Neuropsychopharmacological mechanisms of stimulant drug action in attention-deficit hyperactivity disorder: a review and integration. Behav Brain Res 94: 127-152.

Sonuga-Barke EJS (2002). Psychological heterogeneity in AD/ HD - a dual pathway model of behaviour and cognition. Behav Brain Res 130: 29-36.

Soubrié P (1986). Reconciling the role of central serotonin neurons in human and animal behavior. Behav Brain Sci 9: 319-364.

Swann AC, Bjork JM, Moeller FG, Dougherty DM (2002). Two models of impulsivity: relationship to personality traits and psychopathology. Biol Psychiatry 51: 988-994.

Tomie A (1996). Locating reward cue at response manipulandum (CAM) induces symptoms of drug abuse. Neurosci Biobehav Rev 20: 505-535.

Tomie A, Aguado AS, Pohorecky LA, Benjamin D (1998a). Ethanol induces impulsive-like responding in a delay-of-reward operant choice procedure: impulsivity predicts autoshaping. Psychopharmacology 139: 376-382.

Tomie A, Cunha C, Mosakowski EM, Quartarolo NM, Pohorecky LA, Benjamin D (1998b). Effects of ethanol on Pavlovian autoshaping in rats. Psychopharmacology 139: 154-159.

Volkow ND, Fowler SJ (2000). Addiction, a disease of compulsion and drive: involvement of the orbitofrontal cortex. Cereb Cortex 10: $318-325$.

Williams DR, Williams H (1969). Automaintenance in the pigeon: sustained pecking despite contingent non-reinforcement. J Exp Anal Behav 12: 511-520.

Winstanley CA, Theobald DE, Dalley JW, Robbins TW (2003). Global 5-HT depletion attenuates the ability of amphetamine to decrease impulsive choice in rats. Psychopharmacology 170: 320-331.

Wogar MA, Bradshaw CM, Szabadi E (1993). Effects of lesions of the ascending 5-hydroxytryptaminergic pathways on choice between delayed reinforcers. Psychopharmacology 111: 239-243. 